\title{
CLINICAL PROFILE OF MALIGNANT PLEURAL EFFUSIONS IN A TERTIARY CARE CENTRE
}

\author{
Reshmi Sasidharan Nair ${ }^{1}$
}

${ }_{1}^{1}$ Associate Professor, Department of Pulmonary Medicine, GMC, Trivandrum, Kerala, India.

\section{BACKGROUND}

\section{ABSTRACT}

Malignant pleural effusions are an important cause of exudative pleural effusions we see in clinical practice. The other main causes being syn-pneumonic and tuberculosis. The clinical features of the patient, and pleural fluid characteristics help us differentiate the cause in majority of cases. Malignant effusions are mostly lymphocytic and so the major differential is a tuberculous effusion, which is common in our setting. The former usually occurs as a far advanced disease, whereas the latter is often curable. Here comes the importance of identifying the aetiology of the effusion.

The aim of this study was to determine the clinical features of the patient, and pleural fluid characteristics of malignant pleural effusion cases attending a tertiary care centre and to determine the mode of diagnosis in these cases.

\section{MATERIALS AND METHODS}

Setting and Design- This was a descriptive study done in the Department of Pulmonary Medicine, GMC, Trivandrum for six months from November 2017. All cases of pleural effusion attending the department during the period were evaluated to determine the aetiology and cases with malignant pleural effusions were included in the study.

Methodology- A case was diagnosed as malignant if a cytological/histopathological evidence of malignancy was obtained from pleural fluid or any extrapleural site. If pleural fluid results were negative, a thoracoscopy and pleural biopsy was done. Even after these, if a positive pathology report was not obtained, they were still taken as malignancy if the radiological picture was very much suggestive. 50 consecutive cases of malignant pleural effusion were included in the study. The demographic details, smoking habits and pleural fluid characteristics were studied in all cases.

Statistical Analysis was done using Epi Info 7. Quantitative variables were expressed as means \pm SD and categorical variables as proportion $(95 \%, \mathrm{CI})$.

\section{RESULTS}

$62 \%$ of cases were males. Mean age was 62.08 (SD 13.83). 16\% were current smokers. All were exudative effusions with a mean lymphocyte count of 81.12 (SD- 19.38). Pleural fluid cytology was positive in $62 \%$ and the commonest histology was adenocarcinoma.

\section{CONCLUSION}

Malignant pleural effusions are mostly exudative lymphocytic effusions. Usual aetiology is adenocarcinoma and pleural fluid cytology is yielding in majority.

\section{KEY WORDS}

Clinical Profile, Pleural Fluid, Pleural Effusion, Malignant.

HOW TO CITE THIS ARTICLE: Nair RS. Clinical profile of malignant pleural effusions in a tertiary care centre. J. Evolution Med. Dent. Sci. 2018;7(31):3472-3475, DOI: 10.14260/jemds/2018/782

\section{BACKGROUND}

Pleural effusions are a common disease presentation in routine clinical practice, especially for a pulmonologist. These are mainly classified as transudative and exudative, based on the Light's criteria. Transudative effusions are mostly secondary to cardiac failure, cirrhosis liver, hypoproteinaemia etc. Unless there is clinical confusion in diagnosis or the patient is symptomatic with severe breathlessness, there is no need to aspirate such effusions and do fluid studies. This is not the case with exudative pleural effusions, where finding out the aetiology is crucial in diagnosing and treating the patient.

'Financial or Other Competing Interest': None.

Submission 18-06-2018, Peer Review 16-07-2018,

Acceptance 23-07-2018, Published 30-07-2018.

Corresponding Author:

Dr. Reshmi Sasidharan Nair,

Associate Professor,

Department of Pulmonary Medicine,

GMC, Trivandrum, Kerala, India.

E-mail: reshmisathish@rediffmail.com

DOI: $10.14260 /$ jemds $/ 2018 / 782$
Common aetiologies of exudative effusions are synpneumonic, malignant, tuberculous etc. Diagnostic dilemma often occurs with the latter two since they are lymphocytic effusions. Diagnosis is made with the clinical features and pleural fluid characteristics usually. If not yielding, invasive procedures like thoracoscopy and pleural biopsy are done. In indicated cases, a bronchoscopy might also help in reaching the diagnosis. A quarter of all pleural effusion cases and 30\% to $70 \%$ of all exudative pleural effusions presenting to hospitals are secondary to malignancy.(1)

Pleural fluid cytology is diagnostic in more than twothirds of cases of malignant pleural effusion. Several reports give positivity rates ranging from $60 \%$ to $90 \%$. The common primary tumours which can metastasise to pleura are lung, breast, ovary, lymphoma, gastrointestinal tumours etc. When pleural metastasis occurs, tumour cells 'seed' the mesothelial surface of pleura or invade the subserous layer. In the former case there will be abundant tumour cells in pleural fluid, whereas in the latter there is paucity of malignant cells in the fluid. Adenocarcinoma lung is the commonest to invade pleura. Immunohistochemistry of pleural fluid helps identify the primary in some cases. $(2,3)$ 
Pleural effusions associated with malignancy, but without any direct involvement of tumour in the pleura are called paramalignant effusions. These are usually transudates and will not have any demonstrable malignant cells in pleural fluid or pleural tissue. Common causes are lymphatic obstruction, bronchial obstruction, complications of chemo and radiotherapy, hypoalbuminaemia etc. $(2,3)$

Malignant pleural effusions are usually lymphocytic (50\%-70\%), but the percentage of lymphocytes will be lesser than in tuberculous effusions ( $>80 \%)$. Presence of mesothelial cells also vary. These will be abundant in the initial phase of pleural infiltration, but decreases later in advanced stages with pleural fibrosis. $(2,3)$

Malignant pleural fluid is usually an exudate. If the fluid is exudate as per LDH criteria and not with protein criteria, a malignant aetiology is to be ruled out. $<5 \%$ of effusions associated with malignancy can be transudate also.(2,3)

This study was done to determine the clinical and pleural fluid characteristics of diagnosed cases of malignant pleural effusions in a tertiary care centre. The method of diagnosing malignancy in these cases were also studied.

\section{MATERIALS AND METHODS}

This was a descriptive study done in the Department of Pulmonary Medicine, Government Medical College, Trivandrum, for a period of six months from November 2017. All cases of pleural effusion attending the department during the study period were evaluated to determine the aetiology and cases with a malignant aetiology were included in the study. A case was diagnosed as malignant if there is cytological or histopathological evidence of malignancy in pleural fluid/ pleural tissue or from an extrapleural site. In cases where the pleural fluid results were inconclusive, a thoracoscopy and pleural biopsy was done. Even when all these investigations were negative for malignancy, a diagnosis of malignancy was still made in a few cases when the radiological findings along with the clinical picture very much suggested such a diagnosis. 50 consecutive cases of malignant effusion were taken for the study. The demographic details, smoking habits including exposure to kitchen smoke and pleural fluid characteristics were studied in all. The mode of diagnosing malignancy in each case was also noted.

\section{Statistical Analysis}

Was done using Epi Info 7 after entering the data in Microsoft Excel. Quantitative variables were expressed as means \pm SD and categorical variables as proportion $(95 \%$ confidence limits).

\section{RESULTS}

Of the 50 patients studied, 31 were males (62\%). The demographic details and smoking habits of the study population are detailed in Table 1 . Mean age of the study population was 62.08 years (SD 13.83) and the median age was 64.5 years $(52-70.5)$.

\begin{tabular}{|c|c|c|c|c|c|}
\hline & & & & 95\% & o CI \\
\hline Baseline Chara & cteristics & No. & $\%$ & Lower & Higher \\
\hline Sex & Female & 19 & $38.00 \%$ & $24.65 \%$ & $52.83 \%$ \\
\hline & Male & 31 & $62.00 \%$ & $47.17 \%$ & $75.35 \%$ \\
\hline Smoking & Current & 8 & $16.00 \%$ & $7.17 \%$ & $29.11 \%$ \\
\hline & Past & 15 & $30.00 \%$ & $17.86 \%$ & $44.61 \%$ \\
\hline & Never & 27 & $54.00 \%$ & $39.32 \%$ & $68.19 \%$ \\
\hline Smoking Index & $<400$ & 14 & $60.87 \%$ & $38.54 \%$ & $80.29 \%$ \\
\hline & $>400$ & 9 & $39.13 \%$ & $19.71 \%$ & $61.46 \%$ \\
\hline Kitchen Smoke & $\mathrm{N}$ & 24 & $48.00 \%$ & $33.66 \%$ & $62.58 \%$ \\
\hline & $\mathrm{Y}$ & 26 & $52.00 \%$ & $37.42 \%$ & $66.34 \%$ \\
\hline & & & & Dation & \\
\hline
\end{tabular}

The pleural fluid characteristics of the patients studied are summarised in Table 2.

\begin{tabular}{|c|c|c|c|}
\hline Pleural Fluid & Mean & SD & Median (25\% - 75\%) \\
\hline Lymphocyte \% & 81.12 & 19.38 & $84(70.5-99.5)$ \\
\hline Protein (g\%) & 4.62 & 0.87 & $4.7(4-5.2)$ \\
\hline Sugar (mg\%) & 109.31 & 62.35 & $101(73-139.5)$ \\
\hline ADA (IU/L) & 16.16 & 10.44 & $12.2(9-19.9)$ \\
\hline \multicolumn{2}{|c|}{ Table 2. Pleural Fluid Characteristics of Patients } \\
\hline
\end{tabular}

All patients studied had exudative pleural effusion. The mean ADA (adenosine deaminase) value was $16.16 \pm 10.44$ IU/L. Pleural fluid cytology was positive for malignant cells in $62 \%$ of cases (95\% CI 47.17 - 75.35\%). The commonest histology identified was adenocarcinoma proven in 20 cases. Three cases had lymphoma and one patient was diagnosed as multiple myeloma. The histological pattern in all cases is represented in Figure 1. The split-up of diagnosis in the group 'others' is given in Figure 2. In six patients, we did not get a histological diagnosis even after thoracoscopy and biopsy. A combined clinical and radiological diagnosis of malignancy was made in these cases.

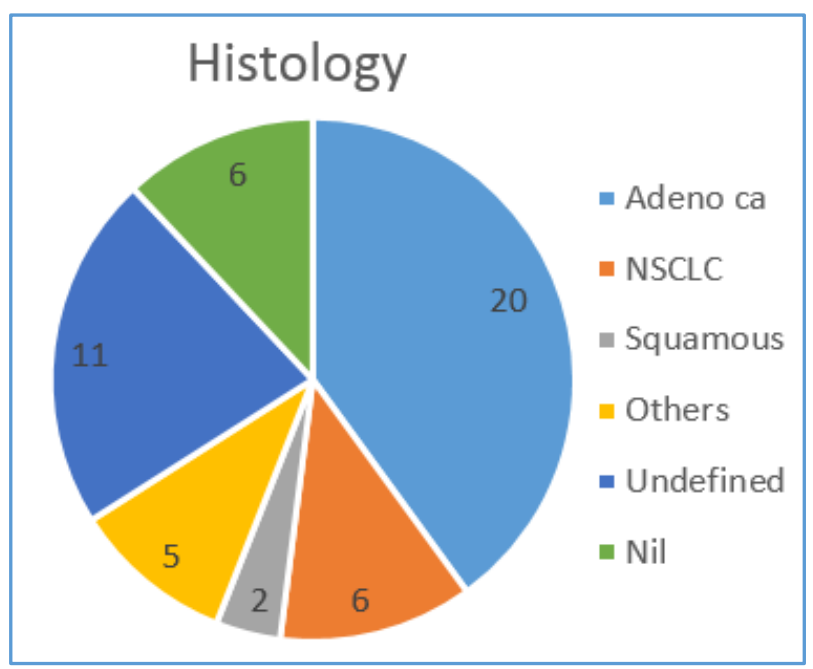

Figure 1 


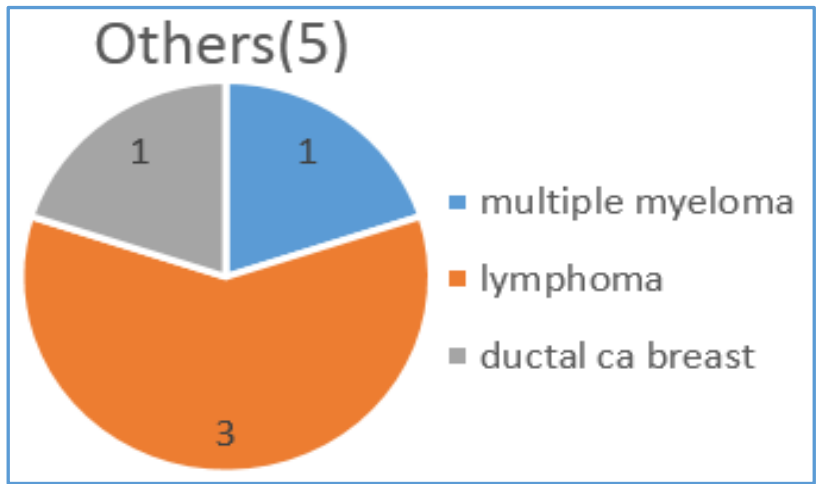

Figure 2

The diagnosis of malignancy was made with pleural fluid cytology in majority of cases (62\%, 95\% CI 47.17 - 75.35\%). Other modalities like aspiration cytology/ biopsy of lung/ lymph node, thoracoscopy, bronchoscopy and biopsy etc. also yielded the diagnosis in some. Table 3 shows the mode of diagnosis in all the cases studied.

\begin{tabular}{|c|c|}
\hline Mode of Diagnosis & Number \\
\hline Pleural fluid cytology & 31 \\
\hline Lymph node biopsy & 2 \\
\hline FNA lung & 3 \\
\hline FNA breast & 1 \\
\hline Lung biopsy & 1 \\
\hline Bronchoscopic biopsy & 1 \\
\hline Thoracoscopic biopsy & 5 \\
\hline Clinical and Radiological & 6 \\
\hline \multicolumn{2}{|c|}{ Table 3. Mode of Diagnosis in the Patients } \\
\hline
\end{tabular}

Four of the cases studied had positive diagnosis from more than one investigation. Pleural fluid cytology and aspiration cytology of metastatic lymph node were positive in 3 cases studied. One case had a positive fluid cytology and malignancy proven from aspiration cytology of lung mass too. A medical thoracoscopy and pleural biopsy were done in 8 patients, of which a positive diagnosis of malignancy was obtained in 5 (62.5\%, 95\% CI 24.49 - 91.48\%).

\section{DISCUSSION}

In this study, 50 consecutive cases of malignant pleural effusion were studied. 31 of them were males (M: F 1.6: 1). Zay Soe and colleagues in a similar study done on malignant pleural effusions have noted a M: F of 1.4: 1.(4) Male preponderance was also seen in other studies (Nitin Gadewad et al).(5) However, Smit Janrao et al in a hospital-based observational study on 89 patients reported almost equal occurrence of malignant pleural effusion in males and females (1.02: 1).(6) The mean age of the study population was $62.08 \pm 13.83$ in our study and $46 \%$ of them were having a history of smoking. The mean age was comparable in other studies also. But a study conducted in Myanmar has reported $82.2 \%$ of ever smokers among the study group, which is much higher than our finding.(4) The presence of malignant pleural effusion usually signals incurability and a poor quality of life for the patient. It is a known factor that smoking is a risk factor for lung carcinoma. Smoking triggers mutagenesis, initiation and progression of lung cancer. But it is not clearly known if cigarette smoke affects pleural malignancy and malignant pleural effusions. This possibility is likely due to the pro-inflammatory and pro-angiogenic properties of smoke. In experimental studies conducted in mice, Sophia Magkouta et al has stated that cigarette smoke promoted malignant pleural effusion formation by enhancing tumour associated inflammation.(7)

There are various reports on the yield of pleural fluid cytology in malignant pleural effusions. These range from $62 \%$ to $90 \% .(8,9)$ According to $\mathrm{KC}$ Ong et al, accuracy of pleural fluid cytology in diagnosing malignancy in pleural effusion ranges from $40 \%$ to $87 \% .^{(10)}$ The pleural disease guideline published by the British Thoracic Society in 2010 says that the sensitivity of pleural fluid cytology for malignancy is around $60 \%$ and there is a $15 \%$ increase with a second procedure. Yield of fluid cytology also depends on the underlying primary tumour, sample preparation and experience of the cytologist.(11) In the current study pleural fluid cytology was positive in $62 \%$ cases, which is similar to the previous observations. In cases where pleural metastasis occurs, there is tumour cell seeding of the mesothelial surface of pleura. There can be invasion in subserous layer also. Mesothelial surface involvement gives abundant tumour cells in pleural fluid.

Immunohistochemistry of pleural fluid cells helps in identifying malignant cells. Here monoclonal antibodies are used against antigens which are unique to benign mesothelial cells, adenocarcinoma cells, malignant mesothelial cells etc. For adenocarcinoma, the antigens targeted are CEA, TTF-1, B 72.3 etc. TTF-1 has high specificity for lung carcinoma. Calretinin, keratin 5/6 etc. are used in mesothelioma. Podoplanin and D2-40 are the best immunohistochemical markers for epithelioid mesothelioma. Electron microscopy of pleural biopsy specimen also helps in making a diagnosis. Histochemical tests can also be employed to differentiate between malignant cells. PAS-D staining if positive, patient is most likely to have adenocarcinoma. Alcian blue stain positivity shows high probability of having mesothelioma.(2)

If the pleural fluid cytology is negative, the next step in the investigation of pleural effusion is doing a pleural biopsy. Though, a blind pleural biopsy may be attempted, it is ideal to do a thoracoscopy to visualise the pleural surface and take biopsy from the affected part. This is the recommendation given in the British Thoracic Society guidelines (2010) for evaluating a pleural effusion also.(12) Thoracoscopy facilities may not be available in all centres, which comes as a major handicap. In our study, thoracoscopy and pleural biopsy were done in 8 cases. A positive yield for malignancy was obtained in 5 of them. The rest had inconclusive results.

Presence of mesothelial cells also vary in pleural fluid. Early in the course of infiltration of pleura, there will be large number of mesothelial cells which decrease in advanced stages with occurrence of pleural fibrosis. Mesothelial cells, especially in their activated form, are often confused with malignant cells. They mimic features of malignant cells. Only an experienced pathologist may be able to differentiate them. Immunohistochemistry also aids in making this distinction. Mesothelial cells are uncommon in tuberculous pleural effusion. In his article on 'cells in pleural fluid,' Light RW et al has observed that only $<1 \%$ of cells in the pleural fluid of tuberculous pleural effusion are comprised of mesothelial cells.(7) 
All of our cases studied were having exudative effusions. This was the finding in many previous studies also. $(4,5,6)$ But literature says around $5 \%$ of malignant pleural effusions can be transudative. $(2,3)$ We had based our diagnosis of exudative effusion on the protein criteria of Light's (pleural fluid protein/ serum protein $>0.5$ ). All patients in the current study did not have the serum and pleural fluid LDH done, so that the LDH ratio can also be calculated. Light RW in his text book on pleural diseases has mentioned that if a pleural effusion is exudative with LDH criteria and not with protein criteria, then a malignant aetiology is to be ruled out.(2)

The major aetiological diagnosis in pleural fluid cytology positive cases was adenocarcinoma in this study, constituting around $52 \%$ (16 out of 31 cases with positive fluid cytology). Adenocarcinoma was the diagnosis in $40 \%$ of all cases, either by cytology or histopathology proof. Of the 20 cases of adenocarcinoma diagnosed, 16 were positive by pleural fluid cytology itself. 2 of them were diagnosed by thoracoscopic pleural biopsy and one each by bronchoscopic biopsy and fine needle aspiration cytology of metastatic lymph node respectively. This was comparable to what has been published earlier by Bhattacharya et al, which also showed a $54 \%$ adenocarcinoma diagnosis among cytology positive malignant effusions.(11)

Zay Soe et al in their study reported a positivity in pleural fluid cytology of $64.4 \%$. However, the commonest diagnosis for them was metastatic large cell carcinoma.(4) Apart from adenocarcinoma, the other diagnosis obtained in this study were non-small cell lung carcinoma and squamous cell carcinoma metastasis. Among the non-lung primary sites, 1 was metastasis from ductal carcinoma breast, 3 were cases of lymphoma and 1 was having multiple myeloma. Literature also supports this fact that around $80 \%$ of all malignant pleural effusions are secondary to carcinoma lung, breast, ovary, gastrointestinal tract and lymphoma. Of these, lung and breast carcinoma account for $65 \%$.

The main differential diagnosis of a lymphocytic pleural effusion is tuberculous effusion. Adenosine Deaminase (ADA) estimation in pleural fluid helps differentiating this from malignant pleural effusions in majority of cases. Whereas ADA levels are high in tuberculosis, malignant effusions are usually low ADA, lymphocytic effusions. JM Porcel et al observed that a higher RBC count and a lower ADA activity in pleural fluid favours a diagnosis of malignancy.(13) In this study, the mean ADA was $16.16 \pm 10.44 \mathrm{IU} / \mathrm{L}$, which is well below the range seen in tuberculous effusions. The mean ADA was $23.83 \mathrm{IU} / \mathrm{L}$ in a similar study done in Myanmar. Mean lymphocyte count of the pleural fluid was $81.12 \pm 19.38 \%$ in our study. Lymphocytic predominance of pleural fluid was noted in previous studies also.(5)

\section{CONCLUSION}

Malignant pleural effusions are seen more in males. These are generally lymphocyte predominant exudative pleural effusions with a low adenosine deaminase level.
Adenocarcinoma is the commonest aetiology observed. Pleural fluid cytology gives a positive result in about twothirds of the cases. Thoracoscopic pleural biopsy helps clinching the diagnosis in cases where fluid cytology is negative. A few cases remain undiagnosed even after these and may need a clinical and radiological correlation to make the final diagnosis.

\section{ACKNOWLEDGEMENT}

I sincerely acknowledge the help given by Dr. Sanjeev Nair, Department of Pulmonary Medicine, Government Medical College, Trivandrum, in doing the statistical analysis of this study.

\section{REFERENCES}

[1] Light RW. Management of pleural effusions. J Formos Med Assoc 2000;99(7):523-31.

[2] Light MD, Richard W. Pleural diseases. $6^{\text {th }}$ edn. Lippincott Williams \& Wilkins 2013.

[3] Fishman JA, Kotloff R, Grippi MA, et al. Fishman's pulmonary diseases and disorders. $5^{\text {th }}$ edn. McGrawHill Education 2015.

[4] Soe Z, Aung Z, Tun KD. A clinical study on malignant pleural effusion. International Journal of Collaborative Research on Internal Medicine \& Public Health 2012;4(5):761-79.

[5] Gadewad N, Deokar K, Ghorpade S. Clinical profile of patients presenting with malignant pleural effusion to a tertiary health care centre. J Assoc Physicians India 2017;65(8):28-31.

[6] Janrao S, Rathod R, Deore P, et al. Clinical profile of patients with malignant pleural effusion. JMSCR 2017;5(6):22905-9.

[7] Magkouta S, Glynos K, Pappas A, et al. Effect of smoking on experimental malignant pleural effusion. European Respiratory Journal 2015;46:PA4343.

[8] Light RW, Erozan YS, Ball WC, et al. Cells in pleural fluid-their value in differential diagnosis. Arch Intern Med 1973;132(6):854-60.

[9] Froudarakis ME. Diagnostic work-up of pleural effusions. Respiration 2008;75(1):4-13.

[10] Ong KC, Indumathi V, Poh WT, et al. The diagnostic yield of pleural fluid cytology in malignant pleural effusions. Singapore Med J 2000;41(1):19-23.

[11] Bhattacharya S, Bairagya TD, Das A, et al. Closed pleural biopsy is still useful in the evaluation of malignant pleural effusion. J Lab Physicians 2012;4(1):35-8.

[12] Hooper C, Lee YC, Maskell N. Investigation of a unilateral pleural effusion in adults: British Thoracic Society Pleural Disease Guideline 2010. Thorax 2010;65 Suppl 2:ii4-17.

[13] Porcel JM, Vives M. Etiology and pleural fluid characteristics of large and massive effusions. Chest 2003;124(3):978-83. 